\title{
Implementation of an objective test of medication adherence in a hypertensive population
}

\author{
Authors: Alexander Lawson, ${ }^{A}$ Awais Hameed ${ }^{B}$ and Indranil Dasgupta ${ }^{B}$
}

\section{Aims}

To develop, implement and audit the performance of an objective liquid chromatography-tandem mass spectrometry (LC-MS/MS) technique for the detection of antihypertensive medications in urine.

\section{Methods}

We have developed a novel LC-MS/MS method for the detection of 23 commonly prescribed antihypertensive medications in urine in a single assay. The method was first applied to the analysis of urine from a validation cohort of 49 individuals who were taking at least one antihypertensive agent in the screening profile to determine their adherence. Then, following implementation, all urine antihypertensive test results, done for uncontrolled hypertension (blood pressure persistently $>140 / 90 \mathrm{mmHg}$ ) between January 2015 and December 2016 at Heartlands Hospital toxicology laboratory, were analysed. Drugs detected were compared to the antihypertensive drugs prescribed. Patients were classified as adherent (all drugs detected), partially adherent ( $\geq 1$ prescribed drugs detected) or non-adherent (no drugs detected). Demographic and clinical data were compared across the three groups.

\section{Results}

The screening method was found to be reproducible, sensitive and specific with the limit of detection ranging between 0.1 and 1.0 $\mu \mathrm{g} / \mathrm{L}$. Sample preparation is rapid ( 30 seconds) and simple, with a total analysis time of 11 minutes. The assay successfully identified the majority of drugs our validation cohort had admitted to taking with drugs not detected indicating non-adherence to prescribed medication, rather than a lack of analytical sensitivity. For our audit following implementation, 335 clinical samples from nine hypertension centres across the UK were received. Fifty-seven per cent of patients audited were non-adherent to prescribed medication with no prescribed drugs detected in $19 \%$ of patients. Non-adherence to antihypertensive medication was significantly associated with total pill burden, number of antihypertensive

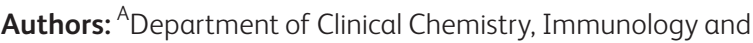

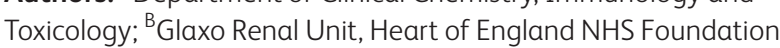
Trust, Birmingham, UK medication prescribed, lower age, female gender, ethnicity, higher blood pressure and higher estimated glomerular filtration rate.

\section{Conclusion}

Non-adherence to prescribed medications is estimated to cost the NHS $£ 100$ per year. Screening of urine specimens for these medications allows a simple, inexpensive ( $€ 30$ per sample), objective test for medication adherence. The data presented here may aid with identification of patients at risk of non-adherence to medication and aid in the development of treatment options for non-adherent patients. The use of techniques such as those developed here may be utilised in other disease areas, allowing for improved patient care and reduced cost in wasted medication.

\section{Conflict of interest statement}

None declared. 